\title{
Association between Diet Quality and Adiposity in the Atlantic PATH Cohort
}

\author{
Vanessa DeClercq ${ }^{1, *}$ (D), Yunsong Cui ${ }^{1}$, Cynthia Forbes ${ }^{1}$, Scott A. Grandy ${ }^{2}$, Melanie Keats ${ }^{2}$, \\ Louise Parker ${ }^{1}$, Ellen Sweeney ${ }^{1}$, Zhijie Michael $\mathrm{Yu}^{1}$ and Trevor J.B. Dummer ${ }^{3}$ \\ 1 Population Cancer Research Program, Department of Pediatrics, Dalhousie University, Halifax, NS B3H 4R2, \\ Canada; yunsong.cui@dal.ca (Y.C.); cynthia.forbes@dal.ca (C.F.); louise.parker@dal.ca (L.P.); \\ ellen.sweeney@dal.ca (E.S.); zhijie.m.yu@gmail.com (Z.M.Y.) \\ 2 School of Health and Human Performance, Dalhousie University, Halifax, NS B3H 1T8, Canada; \\ scott.grandy@dal.ca (S.A.G.); melanie.keats@dal.ca (M.K.) \\ 3 Centre of Excellence in Cancer Prevention, School of Population and Public Health, University of British \\ Columbia, Vancouver, BC V6T 1Z3, Canada; tdummer@mail.ubc.ca \\ * Correspondence: vanessa.de.clercq@dal.ca; Tel.: +1-902-494-4135; Fax: +1-902-494-2089
}

Received: 11 September 2017; Accepted: 18 October 2017; Published: 21 October 2017

\begin{abstract}
The aim of this study was to examine diet quality among participants in the Atlantic Partnership for Tomorrow's Health (PATH) cohort and to assess the association with adiposity. Data were collected from participants $(n=23,768)$ aged 35-69 years that were residents of the Atlantic Canadian provinces. Both measured and self-reported data were used to examine adiposity (including body mass index (BMI), abdominal obesity, waist-to-hip ratio and fat mass) and food frequency questionnaires were used to assess diet quality. Overall, diet quality was statistically different among provinces. Of concern, participants across all the provinces reported consuming only 1-2 servings of vegetables and 1-2 servings fruit per day. However, participants also reported some healthy dietary choices such as consuming more servings of whole grains than refined grains, and eating at fast food restaurants $\leq 1$ per month. Significant differences in BMI, body weight, percentage body fat, and fat mass index were also observed among provinces. Adiposity measures were positively associated with consumption of meat/poultry, fish, snack food, sweeteners, diet soft drinks, and frequenting fast food restaurants, and inversely associated with consumption of whole grains and green tea. Although all four provinces are in the Atlantic region, diet quality vary greatly among provinces and are associated with adiposity.
\end{abstract}

Keywords: diet quality; body mass index; abdominal obesity; fat mass

\section{Introduction}

Life expectancy is lower [1] and the prevalences of most chronic conditions are much higher in the Atlantic region of Canada, including Newfoundland and Labrador, New Brunswick, Nova Scotia, and Prince Edward Island [2,3]. Suboptimal dietary habits have been associated with mortality due to chronic disease [4]. A "Prudent"-type diet (high intake of vegetables, fruits, fish, poultry, whole grains, and low-fat dairy products) has been associated with a lower risk of mortality compared to a "Western"-type diet (characterized by high intake of meat, processed meat, bread, dairy products, coffee, black tea, soft drinks, dressing, sauce, and mayonnaise) [5,6].

Poorer diet quality has been shown to increase the risk of many chronic conditions [7-9]. Studying overall diet quality rather than isolated nutrients to characterize a population's dietary intake has gained wide acceptance in nutritional epidemiology. Diet quality can be assessed by several different indices, with lower scores (i.e., less healthy diets) associated with significantly higher incidences of cancer, diabetes, cardiovascular disease, and mortality [9]. Furthermore, with a 
well-established link between these conditions and obesity, there is a need to focus efforts on dealing with the global rise in obesity [10].

Many variables contribute to obesity including environmental, genetic, and lifestyle factors such as sleep duration, physical inactivity and poor diet [11,12]. A recent systematic review by Asghari et al. reported an inverse association between body mass index (BMI) and diet quality, as assessed by the healthy eating index (HEI) [13]. The HEI and its newer revised versions are methods to assess diet quality based on the Dietary Guidelines for Americans [14] and have been adapted to correspond with Canada's Food Guide $[15,16]$. The Atlantic region of Canada is an area of the country with particularly high rates of obesity [17]; however, there is very little nutritional epidemiological research examining region-specific diet quality in the area. Therefore, the aim of this study was to assess diet quality among Atlantic Canadian provinces and explore the association with obesity in participants of the Atlantic Partnership for Tomorrow's Health (PATH) cohort.

\section{Materials and Methods}

\subsection{Study Population and Data Collection}

Data for the Atlantic PATH cohort were collected during 2009-2015 from the four Atlantic Canada provinces: New Brunswick, Newfoundland and Labrador, Nova Scotia, and Prince Edward Island. Dietary questionnaire data were collected from 23,768 participants aged 35-69 years. Of these participants, 19,393 also had anthropometric measures required to calculate BMI. Anthropometric measures, including height, weight, and waist and hip circumference, were assessed by research nurses in assessment centers and self-reported by participants as part of the questionnaire data.

\subsection{Assessment of Demographic and Lifestyle Risk Factors}

Demographic and lifestyle data including sex, age, education, smoking, alcohol consumption and physical activity were assessed. The level of education completed was categorized as high school or less, college level, and university level or higher. For smoking behavior, participants were categorized as current smoker, former smoker, and never smoked. For alcohol consumption, participants were classified as an abstainer, occasional drinker ( $<3$ times/month), regular drinker (1-3 times/week), and habitual drinker (4-7 times/week). Binge drinking of alcohol was reported when $\geq 5$ and $\geq 4$ drinks were consumed on the same occasion for men and women, respectively. Participants were categorized into never binge drinking, $<1$ per month, 1-3 times per month, once a week, or $>1$ per week. Levels of physical activity were collected using open-ended questions in the International Physical Activity Questionnaire (IPAQ long form) [18]. Participants were asked to identify the frequency and duration of all vigorous, moderate, and walking physical activity (i.e., inclusive of occupational, domestic and leisure-time) within the last seven days. For all domains, metabolic equivalents of a task (MET) values were calculated and summed to obtain a total physical activity score in MET-minutes/week. In compliance with the IPAQ scoring protocol [19], categorical physical activity scores were defined as inactive, moderately active, and active.

\subsection{Assessment of Obesity and Body Composition}

To classify participants as obese/not obese, measured anthropometric data were used to calculate BMI and waist-to-hip ratio, and when anthropometric indices were not available, self-reported measures were used. BMI was calculated by dividing body weight by height in meters squared. Participants with a BMI of $<18.5,18.5-24.9,25.0-29.9$ and $\geq 30.0 \mathrm{~kg} / \mathrm{m}^{2}$ were considered underweight, normal weight, overweight and obese, respectively [20]. The waist-to-hip ratio was set at $>0.90$ for men and $>0.85$ for women [21]. Abdominal obesity was defined as having a waist circumference $\geq 102 \mathrm{~cm}$ for men and $\geq 88 \mathrm{~cm}$ for women [21,22]. Bioelectrical impedance (Tanita Segmental Body Composition Analyzer) was used to assess body composition for participants who visited an assessment center. 
Fat mass and fat-free mass indices were calculated by dividing fat mass and fat-free mass by height in meters squared, respectively.

\subsection{Assessment of Diet Quality}

Dietary assessments of participants were collected via the food frequency questionnaire at the time of enrollment in the study. The food listed in the questionnaire were categorized into six major groups: (1) fruits and vegetables; (2) dairy products; (3) grains; (4) meats and alternatives; (5) desserts and snacks; and (6) beverages and miscellaneous. Each participant was required to recall food intake over the past 12 months, indicating the frequency with which they usually consumed each item, choosing rarely/never, servings per day, servings per week, or servings per month. Additionally, participants were requested to indicate the number of servings habitually consumed in a typical day for fruits and vegetables, dairy products, grains, meat and alternatives. The food frequency questions were used to calculate a HEI score for each participant, producing a continuous variable with a minimum score of 0 (indicative of poorest dietary habits) and a maximum score of 60 (indicative of optimal dietary habits), as previously described [22].

\subsection{Statistical Analysis}

Data analyses were performed with IBM SPSS Statistics software (version 22, SPSS Inc., Chicago, IL, USA) and a $p$-value of $<0.05$ was considered statically significant. Chi-square analyses were used to determine significant associations between provinces and demographic (sex, age, and education), behavioral (physical activity, drinking, and smoking), and adiposity measures. Categorical variables were presented as counts (\%) and continuous variables were presented as mean \pm standard deviation (SD). Differences in continuous anthropometric data among provinces were analyzed using ANOVA, if necessary followed by post hoc two-tailed $t$ tests adjusted with a Bonferroni correction for multiple comparisons. Spearman's correlation coefficients were used to assess the relationship between BMI and dietary habits. Log-binomial regression models were used to evaluate dietary variables associated with obesity. The association between obesity and each dietary variable are presented as prevalence ratios with $95 \%$ confidence intervals ( $95 \% \mathrm{CI}$ ). For the development of the statistical models for predicting the probability of having a BMI $>30$, both demographic variables and lifestyle behaviors were included in the regression models. Variables with a $p<0.05$ (sex, age, education, smoking, alcohol use, physical activity, and province) were retained in the final regression models.

\section{Results}

\subsection{Adiposity, Demographic and Lifestyle Characteristics}

There were more female than male participants overall, however, there was no statistical difference in sex distribution among the provinces. Most participants were 50-59 years of age. Overall, nearly half $(46 \%)$ of participants have university level education, with a lower percentage in Newfoundland and Labrador (37\%), and Prince Edward Island (38\%). The lifestyle behaviors of smoking, alcohol drinking, and physical activity also statistically differed among provinces. Newfoundland and Labrador (13\%), and Prince Edward Island (11\%) reported the highest percentage of current smokers, Nova Scotia reported the highest habitual drinking $(18 \%)$, and Prince Edward Island reported the highest percent of inactive participants (18\%) (Table 1).

Statistically significant differences in multiple measures of adiposity were also observed among provinces (Table 1). Newfoundland and Labrador, Prince Edward Island, New Brunswick, and Nova Scotia consistently reported highest to lowest for BMI, body weight, percentage body fat, and the fat mass index. This was reflected by Newfoundland and Labrador having the highest, and Nova Scotia having the lowest proportion of participants with a BMI in the obese range ( $\left.\geq 30 \mathrm{~kg} / \mathrm{m}^{2}\right)(59 \% \mathrm{vs} .24 \%)$, abdominal obesity ( $57 \%$ vs. $49 \%$ ) and a waist-to-hip ratio above guidelines ( $68 \%$ vs. $62 \%$ ) (Table 1$)$. 
Table 1. Demographic and behavioral characteristics of Atlantic PATH participants.

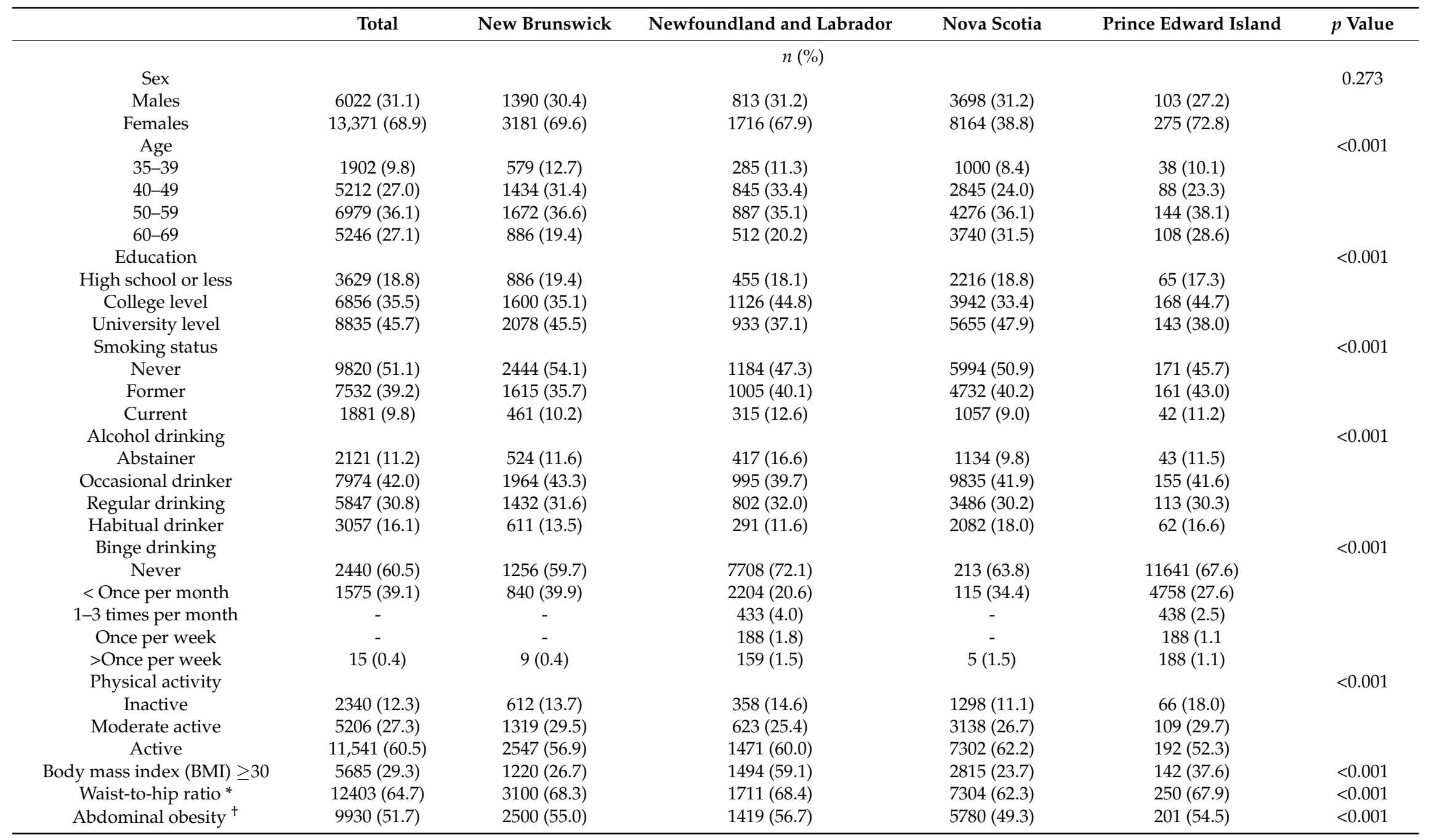


Table 1. Cont

\begin{tabular}{|c|c|c|c|c|c|c|}
\hline & Total & New Brunswick & Newfoundland and Labrador & Nova Scotia & Prince Edward Island & $p$ Value \\
\hline & & & Mean (SD) & & & \\
\hline Height, cm & $166.8(9.7)$ & $166.9(9.3)$ & $166.5(9.1)$ & $166.9(9.0)$ & $166.5(9.8)$ & 0.285 \\
\hline Weight, km & $77.0(16.5)$ & $77.3(15.6)^{b}$ & $83.8(15.2)^{a}$ & $75.4(16.8)^{\mathrm{c}}$ & $79.4(14.8)^{b}$ & $<0.001$ \\
\hline Waist, $\mathrm{cm}$ & $93.5(15.6)$ & $94.3(15.5)^{\mathrm{a}}$ & $94.6(16.3)^{\mathrm{a}}$ & $92.9(15.4)^{b}$ & $94.1(14.9)^{\mathrm{a}, \mathrm{b}}$ & $<0.001$ \\
\hline Hips, cm & $105.0(12.9)$ & $105.3(13.3)^{a, b}$ & $105.7(13.2)^{a}$ & $107.8(12.7)^{b}$ & $105.4(11.3)^{a, b}$ & 0.004 \\
\hline BMI, $\mathrm{kg} / \mathrm{m}^{2}$ & $27.6(5.2)$ & $27.7(4.8)^{c}$ & $30.2(4.7)^{\mathrm{a}}$ & $27.0(5.3)^{\mathrm{d}}$ & $28.6(4.7)^{\mathrm{b}}$ & $<0.001$ \\
\hline Waist-to-hip ratio & $0.89(0.10)$ & $0.90(0.11)^{\mathrm{a}}$ & $0.90(0.12)^{a}$ & $0.89(0.10)^{b}$ & $0.89(0.10)^{a, b}$ & $<0.001$ \\
\hline Percentage body fat, $\%$ & $32.5(8.5)$ & $32.3(7.8)^{b}$ & $37.8(7.1)^{\mathrm{a}}$ & $31.8(8.6)^{\mathrm{b}}$ & $33.6(8.6)^{b}$ & $<0.001$ \\
\hline Fat mass index, $\mathrm{kg} / \mathrm{m}^{2}$ & $9.1(5.9)$ & $8.7(2.9)^{b}$ & $11.8(2.8)^{\mathrm{a}}$ & $8.8(6.6)^{b}$ & $9.4(3.6)^{b}$ & $<0.001$ \\
\hline Fat mass, $\mathrm{kg}$ & $25.0(13.9)$ & $24.2(7.0)^{b}$ & $32.6(6.3)^{\mathrm{a}}$ & $24.2(15.6)^{b}$ & $26.5(7.1)^{b}$ & $<0.001$ \\
\hline Healthy eating index (HEI) & $39.1(8.6)$ & $39.8(8.0)^{\mathrm{a}}$ & $37.8(8.9)^{\mathrm{d}}$ & $39.0(8.7)^{\mathrm{c}}$ & $39.5(7.6)^{a, b}$ & $<0.001$ \\
\hline
\end{tabular}

* Waist to hip ratio $\geq 0.9$ for men and $\geq 0.85$ for women. ${ }^{\dagger}$ Waist circumference $>102 \mathrm{~cm}$ for men and $>88 \mathrm{~cm}$ for women. - Data suppressed due to small cell counts. For continuous variables, values in the same row not sharing the same subscript letter are statistical different $(p<0.05)$. SD, standard deviation.

Table 2. Diet quality for fruit and vegetables, grains, milk, and meat and alternatives.

\begin{tabular}{|c|c|c|c|c|c|c|}
\hline & Total & New Brunswick & Newfoundland and Labrador & Nova Scotia & Prince Edward Island & $p$ Value \\
\hline & & & $n(\%)$ & & & \\
\hline $\begin{array}{c}5 \text { or more serving fruits and } \\
\text { vegetables frequency }\end{array}$ & & & & & & $<0.001$ \\
\hline 0 days per week & $1898(9.8)$ & $525(11.5)$ & $285(11.3)$ & $1033(8.7)$ & 47 (12.7) & \\
\hline 1-2 days per week & $3350(17.3)$ & $831(18.2)$ & $607(24.0)$ & $1817(15.3)$ & $85(22.5)$ & \\
\hline 3-4 days per week & $4692(24.2)$ & $1036(22.7)$ & $681(26.9)$ & $2880(24.3)$ & $82(21.7)$ & \\
\hline$\geq 5$ days per week & $9444(48.7)$ & $2175(47.6)$ & $956(37.8)$ & $6127(51.7)$ & $164(43.4)$ & \\
\hline Fruit/vegetable juice frequency & & & & & & 0.084 \\
\hline Never & $2844(18.5)$ & $681(18.2)$ & $341(16.8)$ & $1746(18.9)$ & $67(21.9)$ & \\
\hline 3-4 times per week & $3428(22.3)$ & $674(18.0)$ & $392(19.3)$ & $2307(25.0)$ & $45(14.7)$ & \\
\hline$\geq 5$ times per week & $3508(22.9)$ & $861(23.0)$ & $574(28.3)$ & $1986(21.5)$ & $76(24.8)$ & \\
\hline Green vegetable servings & & & & & & $<0.001$ \\
\hline 0 per day & $1610(14.2)$ & $463(10.9)$ & $322(14.4)$ & $775(17.4)$ & $42(12.3)$ & \\
\hline $1-2$ per day & $8815(77.8)$ & $3362(79.1)$ & $1768(78.9)$ & $3386(76.1)$ & $268(78.4)$ & \\
\hline 3-4 per day & $759(6.7)$ & $354(8.3)$ & $130(5.8)$ & $243(5.5)$ & $28(8.2)$ & \\
\hline$\geq 5$ per day & $142(1.3)$ & 71 (1.7) & $22(1.0)$ & $44(1.0)$ & - & \\
\hline
\end{tabular}


Table 2. Cont.

\begin{tabular}{|c|c|c|c|c|c|c|}
\hline & Total & New Brunswick & Newfoundland and Labrador & Nova Scotia & Prince Edward Island & $p$ Value \\
\hline Servings of vegetables & & & & & & $<0.001$ \\
\hline 0 per day & $749(3.9)$ & $101(2.3)$ & $131(5.3)$ & $502(4.3)$ & $10(2.7)$ & \\
\hline $1-2$ per day & $10,117(53.1)$ & $2411(53.7)$ & $1560(63.7)$ & $5920(50.6)$ & $195(52.6)$ & \\
\hline $3-4$ per day & $6506(34.1)$ & $1542(34.4)$ & $637(26.0)$ & $4182(35.7)$ & $131(35.3)$ & \\
\hline$\geq 5$ per day & $1687(8.9)$ & $433(9.7)$ & $123(5.0)$ & $1094(9.4)$ & $35(9.4)$ & \\
\hline Servings of fruit & & & & & & $<0.001$ \\
\hline 0 per day & $1647(8.6)$ & $305(6.8)$ & $203(8.3)$ & $1106(9.5)$ & $24(6.5)$ & \\
\hline $1-2$ per day & $11,241(59.0)$ & $2588(57.7)$ & $1552(63.3)$ & $6841(58.5)$ & $230(62.0)$ & \\
\hline $3-4$ per day & $5240(27.5)$ & $1324(29.5)$ & $592(24.2)$ & $3204(27.4)$ & $111(29.9)$ & \\
\hline Servings of fruit/vegetable juice & & & & & & $<0.001$ \\
\hline 0 per day & $9603(50.4)$ & 2315 (51.6) & $1152(47.0)$ & $5930(50.7)$ & $183(49.3)$ & \\
\hline $1-2$ per day & $8654(45.4)$ & $1999(44.5)$ & $1196(48.8)$ & $5256(44.9)$ & $176(47.4)$ & \\
\hline $3-4$ per day & $667(3.5)$ & $145(3.2)$ & $85(3.5)$ & $425(3.6)$ & $10(2.7)$ & \\
\hline$\geq 5$ per day & $137(0.7)$ & $29(0.6)$ & $18(7.0)$ & $88(0.8)$ & - & \\
\hline $\begin{array}{l}5 \text { or more serving fruit and } \\
\text { vegetables per day }\end{array}$ & & & & & & $<0.001$ \\
\hline No & $7622(40.0)$ & $1740(38.8)$ & 1209 (49.3) & 4505 (38.5) & $144(38.8)$ & \\
\hline Yes & $11,425(60.0)$ & $2746(61.2)$ & $1242(50.7)$ & $782(61.5)$ & $227(61.2)$ & \\
\hline 0 per day & $1224(7.2)$ & $382(8.7)$ & $290(12.3)$ & $517(5.2)$ & $32(8.8)$ & \\
\hline $1-2$ per day & $10,479(61.6)$ & $2780(63.6)$ & $1542(65.3)$ & $5890(59.6)$ & $233(64.6)$ & \\
\hline $3-4$ per day & $4467(26.2)$ & $1016(23.2)$ & $463(19.6)$ & $2897(29.3)$ & $84(23.2)$ & \\
\hline$\geq 5$ per day & $854(5.0)$ & $193(4.4)$ & $65(2.8)$ & $583(5.9)$ & $13(3.6)$ & \\
\hline Servings of refined grains & & & & & & $<0.001$ \\
\hline 0 per day & $6275(38.1)$ & $1523(36.9)$ & $691(30.6)$ & $3910(40.4)$ & $130(37.8)$ & \\
\hline $1-2$ per day & $8903(54.1)$ & $2238(54.2)$ & $1355(60.1)$ & $5100(52.6)$ & $186(54.1)$ & \\
\hline $3-4$ per day & $1087(6.6)$ & $319(7.7)$ & $171(7.6)$ & $570(5.9)$ & $25(7.3)$ & \\
\hline$\geq 5$ per day & $197(1.2)$ & $46(1.1)$ & $38(1.7)$ & $110(1.1)$ & - & \\
\hline Servings of milk and dairy & & & & & & $<0.001$ \\
\hline 0 per day & $687(3.6)$ & $161(3.6)$ & $88(3.6)$ & $419(3.6)$ & $17(4.6)$ & \\
\hline 1 per day & $5323(28.2)$ & $1179(26.3)$ & $714(29.2)$ & $3311(28.7)$ & $96(26.0)$ & \\
\hline 2 per day & $6910(36.6)$ & $1711(38.2)$ & $947(38.7)$ & $4108(35.7)$ & $127(34.4)$ & \\
\hline$\geq 3$ per day & $5952(31.5)$ & $1431(31.9)$ & $697(28.5)$ & $3685(32.0)$ & $129(35.0)$ & \\
\hline Type of milk usually consumed & & & & & & $<0.001$ \\
\hline Homogenized & $592(3.3)$ & $135(3.1)$ & $114(4.9)$ & $330(3.0)$ & $13(3.8)$ & \\
\hline
\end{tabular}


Table 2. Cont.

\begin{tabular}{|c|c|c|c|c|c|c|}
\hline & Total & New Brunswick & Newfoundland and Labrador & Nova Scotia & Prince Edward Island & $p$ Value \\
\hline $2 \%$ cow's milk & $3714(20.6)$ & $1100(25.6)$ & $524(22.3)$ & $1991(18.2)$ & $89(26.2)$ & \\
\hline $1 \%$ cow's milk & $5934(33.0)$ & $1363(31.7)$ & $731(31.1)$ & $3707(33.8)$ & $115(33.8)$ & \\
\hline Skim cow's milk & $6326(35.1)$ & $1354(31.5)$ & $823(35.0)$ & $4034(36.8)$ & $97(28.5)$ & \\
\hline Soy & $597(3.3)$ & $155(3.6)$ & $48(2.0)$ & $385(3.5)$ & $9(2.6)$ & \\
\hline Other & $835(4.6)$ & $188(4.4)$ & $109(4.6)$ & $518(4.7)$ & $17(5.0)$ & \\
\hline Meat/poultry servings & & & & & & $<0.001$ \\
\hline 0 per day & $650(3.4)$ & $119(2.7)$ & $42(1.7)$ & $478(4.1)$ & $9(2.5)$ & \\
\hline 1 per day & $9891(52.4)$ & $2195(49.1)$ & $1300(53.1)$ & $6187(53.6)$ & $182(49.6)$ & \\
\hline 2 per day & $6083(32.2)$ & $1604(35.9)$ & $826(33.7)$ & $3494(30.3)$ & $144(39.2)$ & \\
\hline$\geq 3$ per day & $2252(11.9)$ & $550(12.3)$ & $282(11.5)$ & $1382(12.0)$ & $32(8.7)$ & \\
\hline Egg servings & & & & & & $<0.001$ \\
\hline 0 per day & $2102(11.9)$ & $725(17.8)$ & $364(16.4)$ & $958(8.7)$ & $44(12.9)$ & \\
\hline 1 per day & $4352(24.6)$ & $1343(33.0)$ & $854(38.4)$ & $2022(18.4)$ & $119(35.0)$ & \\
\hline 2 per day & $4805(27.2)$ & $985(24.2)$ & $494(22.2)$ & $3236(29.4)$ & $80(23.5)$ & \\
\hline$\geq 3$ per day & $6428(36.3)$ & $1013(24.9)$ & $513(23.1)$ & $4792(43.5)$ & $97(28.5)$ & \\
\hline Fish servings & & & & & & 0.005 \\
\hline 0 per day & $7455(46.9)$ & $1889(49.0)$ & $909(44.3)$ & $4482(46.6)$ & $149(49.2)$ & \\
\hline 1 per day & $7172(45.2)$ & $1671(43.4)$ & $947(46.2)$ & $4408(45.8)$ & $131(43.2)$ & \\
\hline 2 per day & $1015(6.4)$ & $242(6.3)$ & $157(7.7)$ & $590(6.1)$ & $23(7.6)$ & \\
\hline 0 per day & $12,425(95.1)$ & $3516(94.7)$ & $1873(97.9)$ & $6515(95.0)$ & $287(98.0)$ & \\
\hline 1 per day & $563(4.3)$ & $205(5.5)$ & $35(1.8)$ & $316(4.5)$ & $6(2.0)$ & \\
\hline 2 per day & $44(0.3)$ & $15(0.4)$ & - & $26(0.4)$ & - & \\
\hline$\geq 3$ per day & $18(0.1)$ & - & - & $12(0.2)$ & - & \\
\hline Bean or other legume servings & & & & & & $<0.001$ \\
\hline 0 per day & $8645(57.5)$ & $2308(59.6)$ & $1235(55.5)$ & 4889 (55.5) & $188(63.6)$ & \\
\hline 1 per day & $5718(38.0)$ & $1388(35.8)$ & $695(34.6)$ & $3512(39.9)$ & $110(35.9)$ & \\
\hline 2 per day & $551(3.7)$ & $145(3.7)$ & $65(3.2)$ & $334(3.8)$ & $6(2.0)$ & \\
\hline$\geq 3$ per day & $123(0.8)$ & $34(0.9)$ & $13(0.6)$ & $74(0.8)$ & - & \\
\hline Nut or seed servings & & & & & & $<0.001$ \\
\hline 0 per day & $5842(36.2)$ & $1411(34.7)$ & $980(47.4)$ & $3324(34.5)$ & $111(34.9)$ & \\
\hline 1 per day & $8464(52.5)$ & $2191(54.0)$ & $913(44.2)$ & $5159(53.5)$ & $182(57.2)$ & \\
\hline 2 per day & $1492(9.3)$ & $382(9.4)$ & $145(7.0)$ & $940(9.8)$ & $22(6.9)$ & \\
\hline$\geq 3$ per day & $329(2.0)$ & $77(1.9)$ & $28(1.4)$ & $218(2.3)$ & - & \\
\hline
\end{tabular}

- Data suppressed due to small cell counts. 
Table 3. Diet quality for beverages and miscellaneous intake.

\begin{tabular}{|c|c|c|c|c|c|c|}
\hline & Total & New Brunswick & Newfoundland and Labrador & Nova Scotia & Prince Edward Island & $p$ Value \\
\hline & & & $n(\%)$ & & & \\
\hline Most often eaten on bread & & & & & & $<0.001$ \\
\hline Butter & $5706(29.9)$ & $1360(30.2)$ & $411(16.6)$ & $3768(32.3)$ & $160(42.9)$ & \\
\hline Olive oil & $409(2.1)$ & $54(1.2)$ & $22(0.9)$ & $327(2.8)$ & $5(1.3)$ & \\
\hline Margarine & $6587(34.5)$ & $1567(34.7)$ & $1180(47.6)$ & $3701(31.7)$ & $116(31.1)$ & \\
\hline Low-fat margarine & $4324(22.6)$ & $965(21.4)$ & $631(25.4)$ & $2665(22.8)$ & $50(13.4)$ & \\
\hline None & $2067(10.8)$ & $564(12.5)$ & $237(9.6)$ & $1218(10.4)$ & 42 (11.3) & \\
\hline Frequency of garlic, hot spices, ginger & & & & & & $<0.001$ \\
\hline 0 per week & $2667(13.8)$ & $590(12.9)$ & $498(19.7)$ & $1511(12.7)$ & $68(18.0)$ & \\
\hline 1-2 days per week & $8013(41.3)$ & $2054(45.0)$ & $1191(47.1)$ & $4591(38.7)$ & $154(40.7)$ & \\
\hline 3-4 days per week & $5061(26.1)$ & $1182(25.9)$ & $541(21.4)$ & $3235(27.3)$ & $89(23.5)$ & \\
\hline$\geq 5$ days per week & $3634(18.7)$ & $742(16.2)$ & $297(11.8)$ & $2322(21.3)$ & $67(17.7)$ & \\
\hline Coffee servings & & & & & & $<0.001$ \\
\hline 0 cups per day & $2057(15.2)$ & $622(17.3)$ & $366(21.3)$ & $996(12.6)$ & $66(21.4)$ & \\
\hline$<2$ cups per day & $3442(25.4)$ & $864(24.1)$ & $456(26.5)$ & $2055(26.0)$ & $58(18.1)$ & \\
\hline $2-4$ cups per day & $7468(55.1)$ & $1938(54.0)$ & $832(48.3)$ & $4507(57.0)$ & $171(55.3)$ & \\
\hline$>4$ cups per day & $598(4.4)$ & $164(4.6)$ & $68(3.9)$ & $350(4.4)$ & $14(4.5)$ & \\
\hline Decaffeinated coffee servings & & & & & & $<0.001$ \\
\hline 0 cups per day & $3628(64.9)$ & $1176(75.6)$ & $604(68.6)$ & $1715(57.4)$ & $124(83.2)$ & \\
\hline$<2$ cups per day & $1172(21.0)$ & $233(14.9)$ & $169(19.2)$ & $744(24.9)$ & $20(13.4)$ & \\
\hline $2-4$ cups per day & 754 (13.5) & $145(9.3)$ & $99(11.3)$ & $504(16.9)$ & $5(3.4)$ & \\
\hline$>4$ cups per day & $33(0.6)$ & - & $8(0.9)$ & $23(0.8)$ & - & \\
\hline Black tea servings & & & & & & $<0.001$ \\
\hline 0 cups per day & $2815(31.7)$ & $970(48.4)$ & $440(33.6)$ & $1307(24.5)$ & $91(44.4)$ & \\
\hline$<2$ cups per day & $2334(26.3)$ & $499(24.9)$ & $298(22.7)$ & $1482(27.8)$ & $51(24.9)$ & \\
\hline $2-4$ cups per day & $3225(36.3)$ & $480(24.0)$ & $520(39.7)$ & $2157(40.4)$ & $59(28.8)$ & \\
\hline$>4$ cups per day & $503(5.7)$ & $54(2.7)$ & $53(4.0)$ & $392(7.3)$ & - & \\
\hline Green tea servings & & & & & & $<0.001$ \\
\hline 0 cups per day & $3248(49.8)$ & $1045(57.4)$ & $551(59.2)$ & $1546(43.3)$ & $98(55.1)$ & \\
\hline$<2$ cups per day & $2136(32.8)$ & $538(29.5)$ & $215(23.1)$ & $1322(37.0)$ & $52(29.2)$ & \\
\hline $2-4$ cups per day & $1066(16.2)$ & $224(12.3)$ & $151(16.2)$ & $664(18.6)$ & $24(14.6)$ & \\
\hline$>4$ cups per day & $70(1.1)$ & $14(0.8)$ & $14(1.5)$ & $39(1.1)$ & - & \\
\hline Other tea servings & & & & & & $<0.001$ \\
\hline 0 cups per day & $3171(47.3)$ & $1046(64.1)$ & $491(46.6)$ & 1518 (39.6) & $109(66.9)$ & \\
\hline$<2$ cups per day & $1916(28.6)$ & $377(23.1)$ & $248(23.5)$ & $1254(32.7)$ & $39(23.9)$ & \\
\hline $2-4$ cups per day & $1433(21.4)$ & $194(11.9)$ & $285(27.0)$ & $938(34.5)$ & $13(8.0)$ & \\
\hline$>4$ cups per day & $179(2.7)$ & $14(0.9)$ & $30(2.8)$ & $133(3.5)$ & - & \\
\hline
\end{tabular}


Table 3. Cont

\begin{tabular}{|c|c|c|c|c|c|c|}
\hline & Total & New Brunswick & Newfoundland and Labrador & Nova Scotia & Prince Edward Island & $p$ Value \\
\hline Snack food frequency & & & & & & $<0.001$ \\
\hline Never & 2573 (13.6) & $527(11.5)$ & $308(12.2)$ & $1673(14.7)$ & $57(15.1)$ & \\
\hline 1-2 times per week & $8411(44.4)$ & $2056(45.0)$ & $1090(43.1)$ & $5089(44.6)$ & $157(41.5)$ & \\
\hline 3-4 times per week & $4963(26.2)$ & $1232(27.0)$ & $695(27.5)$ & $2911(25.5)$ & $106(28.0)$ & \\
\hline$\geq 5$ times per week & $2990(15.8)$ & $756(16.5)$ & $434(17.2)$ & $1736(15.2)$ & $58(15.3)$ & \\
\hline Dessert frequency & & & & & & 0.037 \\
\hline Never & $1942(10.0)$ & $477(10.4)$ & $223(8.8)$ & $1194(10.1)$ & $43(11.4)$ & \\
\hline 1-2 times per week & 6485 (33.5) & $1575(34.5)$ & $791(31.3)$ & 3966 (33.5) & $131(34.7)$ & \\
\hline 3-4 times per week & $5000(25.8)$ & $1177(25.8)$ & $687(27.2)$ & $3024(25.5)$ & $98(25.9)$ & \\
\hline$\geq 5$ times per week & $5953(30.7)$ & $1339(29.3)$ & $827(32.7)$ & $3669(31.0)$ & $106(28.0)$ & \\
\hline Artificial sweetener use & & & & & & $<0.001$ \\
\hline Never & $15,816(86.3)$ & $3616(85.5)$ & $1860(78.1)$ & $10,027(88.2)$ & $313(90.5)$ & \\
\hline $1 / 4$ of the time & $393(2.1)$ & $97(2.3)$ & $60(2.5)$ & $233(2.0)$ & - & \\
\hline $1 / 2$ of the time & $252(1.4)$ & $58(1.4)$ & $52(2.2)$ & $140(1.2)$ & - & \\
\hline $3 / 4$ of the time & $166(0.9)$ & $48(1.1)$ & $24(1.0)$ & $92(0.8)$ & - & \\
\hline Always & $1701(9.3)$ & $408(9.7)$ & $385(16.2)$ & $882(7.8)$ & $26(7.5)$ & \\
\hline Regular soft drink frequency & & & & & & $<0.001$ \\
\hline Never & $6596(37.1)$ & $1387(33.5)$ & $726(33.4)$ & $4351(39.2)$ & $110(33.4)$ & \\
\hline 1-3 times per month & $7342(41.2)$ & $1833(44.2)$ & $814(37.5)$ & $4537(40.8)$ & $145(44.1)$ & \\
\hline 1-4 times per week & $2620(14.7)$ & $608(14.7)$ & $349(16.1)$ & $1612(14.5)$ & $45(13.7)$ & \\
\hline 5-7 times per week & $613(3.4)$ & $162(3.9)$ & $140(6.4)$ & $292(2.6)$ & $17(5.2)$ & \\
\hline$>1$ per day & $629(3.5)$ & $153(3.7)$ & $143(6.6)$ & $319(2.9)$ & $12(3.6)$ & \\
\hline Diet soft drink frequency & & & & & & $<0.001$ \\
\hline Never & $7778(48.8)$ & 1795 (50.5) & $739(39.4)$ & $5111(50.0)$ & $133(45.7)$ & \\
\hline 1-3 times per month & $4762(29.8)$ & $1046(29.4)$ & $575(30.7)$ & $3036(29.7)$ & $94(32.3)$ & \\
\hline $1-4$ times per week & $1898(11.9)$ & $357(10.0)$ & $236(12.6)$ & $1266(12.4)$ & $34(11.7)$ & \\
\hline 5-7 times per week & $681(4.3)$ & $185(5.2)$ & $142(7.6)$ & $340(3.3)$ & $13(4.5)$ & \\
\hline$>1$ per day & $837(5.2)$ & $173(4.9)$ & $183(9.8)$ & $461(4.5)$ & $17(5.8)$ & \\
\hline Amount of regular or diet soft drink & & & & & & $<0.001$ \\
\hline$<12$ ounces $(<1$ can/bottle) & $7531(58.8)$ & 1754 (55.5) & $1062(59.2)$ & $4544(60.2)$ & $151(559)$ & \\
\hline $12-16$ ounces ( 1 can/bottle) & 4975 (38.9) & $1328(42.0)$ & $689(38.4)$ & $2834(37.6)$ & $117(43.3)$ & \\
\hline$>16$ ounces $(>1$ can/bottle) & $295(2.3)$ & $81(2.6)$ & $44(2.5)$ & $166(2.2)$ & - & \\
\hline Soy or fish sauce frequency & & & & & & $<0.001$ \\
\hline Never & $8104(42.1)$ & $1737(38.1)$ & $1153(46.1)$ & $5019(42.6)$ & $170(45.1)$ & \\
\hline Rarely & $7360(38.2)$ & $1847(40.5)$ & $851(34.0)$ & $4496(38.2)$ & $146(38.7)$ & \\
\hline Sometimes & $3650(18.9)$ & $936(20.5)$ & $473(18.9)$ & $2174(18.5)$ & $60(15.9)$ & \\
\hline Always & $150(0.8)$ & $37(0.8)$ & $25(1.0)$ & $87(0.7)$ & - & \\
\hline
\end{tabular}


Table 3. Cont.

\begin{tabular}{|c|c|c|c|c|c|c|}
\hline & Total & New Brunswick & Newfoundland and Labrador & Nova Scotia & Prince Edward Island & $p$ Value \\
\hline Salt seasoning frequency & & & & & & $<0.001$ \\
\hline Never & $6996(36.3)$ & $1621(35.5)$ & $1107(44.1)$ & $4098(34.7)$ & $149(39.5)$ & \\
\hline Rarely & $5934(30.8)$ & $1325(29.0)$ & $800(31.9)$ & $3684(31.2)$ & $111(29.4)$ & \\
\hline Sometimes & $3685(19.1)$ & $908(19.9)$ & $387(15.4)$ & $2329(19.7)$ & $55(14.6)$ & \\
\hline Always & $2682(13.9)$ & $708(15.5)$ & $214(8.4)$ & $1687(14.3)$ & $62(16.4)$ & \\
\hline Fast food restaurant frequency & & & & & & $<0.001$ \\
\hline Never & $1372(7.7)$ & $169(4.7)$ & $80(3.5)$ & $1068(9.7)$ & $26(7.6)$ & \\
\hline$\leq 1$ per month & $8306(46.7)$ & $1799(43.5)$ & $981(43.1)$ & $5346(48.7)$ & $157(45.8)$ & \\
\hline $2-4$ times per month & $7365(41.4)$ & $1913(46.3)$ & $1101(48.3)$ & $4184(38.1)$ & $144(42.0)$ & \\
\hline 2-4 times per week & $573(3.2)$ & $169(4.1)$ & $93(4.1)$ & $296(2.7)$ & $13(3.8)$ & \\
\hline$\geq 5$ times per week & $171(1.0)$ & $54(1.3)$ & $23(1.0)$ & $91(0.8)$ & - & \\
\hline Fat/oil most often used & & & & & & $<0.001$ \\
\hline None & $134(0.7)$ & $23(0.5)$ & $24(0.9)$ & $82(0.7)$ & - & \\
\hline Margarine & $815(4.2)$ & $249(5.4)$ & $78(3.1)$ & $468(4.0)$ & $19(5.0)$ & \\
\hline Butter & $210(1.1)$ & $58(1.3)$ & $9(0.4)$ & $133(1.1)$ & $9(2.4)$ & \\
\hline Other & $803(4.1)$ & $170(3.7)$ & $131(5.2)$ & $489(4.1)$ & $10(2.6)$ & \\
\hline Olive oil & $4429(22.8)$ & $1106(24.2)$ & $621(24.6)$ & $2607(22.0)$ & $78(20.6)$ & \\
\hline Canola & $1341(6.9)$ & $245(5.4)$ & $264(10.4)$ & $808(608)$ & $21(5.6)$ & \\
\hline Combination of above & $11,630(60.0)$ & $2720(59.5)$ & $1402(55.4)$ & $7244(61.2)$ & $238(63.0)$ & \\
\hline
\end{tabular}

- Data suppressed due to small cell counts. 


\subsection{Diet Quality}

The average HEI score was 39.1 for the whole region and this was statistically different among provinces with Newfoundland and Labrador receiving the lowest score (37.8) and New Brunswick receiving the highest score (39.8) (Table 1). Fruit and vegetable intake were statistically different among provinces (Table 2). Overall, most participants reported consuming five or more servings of fruits and vegetables on five or more days per week, with the highest percentage in Nova Scotia (52\%) and the lowest in Newfoundland and Labrador (38\%). However, most participants only reported consuming 1-2 servings of vegetables (53\%) and 1-2 servings of fruit per day (59\%) (Table 2). Newfoundland and Labrador reported the lowest percentage of individuals who consumed five or more servings of fruit and vegetables per day ( $50 \%$ in Newfoundland versus $61 \%$ in other provinces).

The intake of grains, milk and dairy, and meat and alternatives varied statistically among provinces. In general, most participants (62\%) reported consuming 1-2 servings of whole grains and $54 \%$ reported consuming 1-2 servings of refined grains per day (Table 2). Most participants (68\%) reported consuming two or more servings of milk per day and skim milk was the most commonly reported type of milk consumed (Table 2). Overall, the majority of participants reported one serving of meat/poultry per day, and New Brunswick and Nova Scotia had the highest percentage of participants $(12 \%)$ consuming three or more servings per day (Table 2). Nova Scotia also had the highest percentage of participants (44\%) consuming three or more servings of eggs per day (Table 2). Most participants $(91 \%)$ reported either none or one serving of fish per day, with Newfoundland and Nova Scotia more likely to consume one serving per day (Table 2). Tofu and bean curd were not commonly consumed among participants ( $<6 \%$ consuming daily), with the lowest rates reported in participants from Newfoundland and Labrador, and Prince Edward Island, and highest in participants from Nova Scotia and New Brunswick (Table 2). Regionally, most participants did not consume beans or legumes daily. Prince Edward Island reported the lowest servings (36\% consumed daily) and Nova Scotia and New Brunswick the highest (45\% consumed daily) (Table 2). In total, most participants (64\%) consumed at least one serving of nuts or seeds per day, except in Newfoundland and Labrador where daily consumption was much less (53\%) (Table 2).

Beverage (soft drink, coffee, and tea) consumption and frequency of garlic, hot spices, and ginger were statistically different among the four provinces (Table 3). Daily soft drink consumption was most prevalent whereas daily coffee consumption was less common in Newfoundland and Labrador. Consumption of garlic, hot spices, and ginger on more than five days per week was most common in Nova Scotia. Forty-five percent and $34 \%$ of participants reported consuming snack foods and desserts 1-2 times per week, respectively, whereas $36 \%$ of participants reported never using salt and $47 \%$ reported visiting fast food restaurants $\leq 1$ per month (Table 3$)$. Overall, the majority $(60 \%)$ of participants used a combination of two or more fats/oil, with olive oil and canola being the most common combination, and margarine being most commonly used on bread (Table 3).

\subsection{Relationship between Adiposity and Dietary Habits}

Servings of meat/poultry, refined grains, coffee, snack food, sweetener use, soft drinks, and frequency of eating at fast food restaurants were all positively associated with BMI. In contrast, the frequency of garlic, hot spices, and ginger; five or more serving of fruit and vegetables; whole grains; tofu; nut/seeds; green tea; green vegetables; all vegetables; fruit, juice; and the HEI were all inversely associated with BMI (Table 4). Waist circumference and percentage body fat were also positively associated with meat/poultry, fish, snack foods, sweeteners, diet soft drinks, and fast-food, and negatively with whole grains (Table 4).

Since there were clear differences in diet quality and adiposity measures among provinces, regression models were used to identify dietary variables that were predictive of obesity (or an obese BMI). Several dietary factors were found to be statistically associated with an obese BMI. In the unadjusted model, participants consuming higher servings of meat/poultry and refined grains were more likely to be obese (Table 5). By contrast, participants were less likely to be obese with 
increased servings of green vegetables, whole grains, tofu, nuts/seeds, vegetables and fruits (Table 5). Participants with a higher score on the HEI were less likely to be obese (Table 5). All relationships, with the exception of green vegetables, remained statistically significant after adjusting for additional co-variables (Table 5). By controlling for the co-variables, the regression models enabled us to test the strength of the associations between the exposure variables (diet quality) and study outcomes (obesity), and the chi-square goodness of fit test indicated that the model was a good fit $(p>0.05)$.

Table 4. Association between BMI and frequency/servings of dietary variables.

\begin{tabular}{|c|c|c|c|}
\hline Variable & $R$ & Variable & $\boldsymbol{R}$ \\
\hline Frequency of garlic, hot spices, ginger & $-0.048^{*, \dagger}$ & Servings of green tea & $-0.029 *,+, \S$ \\
\hline Frequency of $\geq 5$ fruit and vegetables & $-0.081^{*, \dagger}$ & Servings of other tea & $0.024^{t, \S}$ \\
\hline Servings of green vegetables & $-0.032 *+$ & Frequency of snack food & $0.091 *,+, \S$ \\
\hline Servings of whole grains & $-0.059 *,+\S$ & Frequency of desserts & $0.005^{t, \S}$ \\
\hline Servings of refined grains & $0.075^{*, \dagger}$ & Frequency of sweetener use & $0.101 *,+, \S$ \\
\hline Servings of milk products & $0.013 \S$ & Frequency of fruit juice & $-0.042 *, \S$ \\
\hline Servings of eggs & $-0.012 \S$ & Frequency of regular soft drink & $0.111^{*,+}$ \\
\hline Servings of meat/poultry & $0.106^{*,+, \S}$ & Frequency of diet soft drink & $0.170^{*, t, \S}$ \\
\hline Servings of fish & $0.018 *,+, \S$ & Frequency of soy sauce & $-0.005 \S$ \\
\hline Servings of tofu & $-0.050 *,+$ & Frequency of seasoning with salt & $0.003^{+}$ \\
\hline Servings of beans & -0.011 & Frequency of fast food restaurant & $0.162 *,+, \S$ \\
\hline Servings of nuts/seeds & $-0.058^{*, \dagger}$ & Servings of vegetables & $-0.069^{*,+}$ \\
\hline Servings of coffee & $0.032 *,+$ & Servings of fruit & $-0.051^{*, \dagger}$ \\
\hline Servings of decaffeinated coffee & $0.012^{+, \S}$ & Servings of fruit/vegetable juice & $-0.015 *, \S$ \\
\hline Servings of black tea & $0.008^{+, \S}$ & HEI & $-0.073 *,+\S$ \\
\hline
\end{tabular}

Results are expressed as Spearman's correlation coefficients for associations with BMI. * Indicates statistically significant association with BMI $(p<0.05)$. Indicates statistically significant association found with waist circumference $(p<0.05)$, coefficients not shown. $\S$ Indicates statistically significant association found with percentage body fat $(p<0.05)$, coefficients not shown.

Table 5. Regression models for servings of dietary components as predictors of obesity ${ }^{\dagger}$.

\begin{tabular}{|c|c|c|c|}
\hline Variable & $\begin{array}{c}\text { Prevalence Ratio } \\
\text { (95\%CIs) }\end{array}$ & Variable & $\begin{array}{c}\text { Prevalence Ratio } \\
\text { (95\%CIs) }\end{array}$ \\
\hline Servings of green vegetables & & Servings of nuts/seeds & \\
\hline Unadjusted & $0.95(0.92-0.98)$ & Unadjusted & $0.90(0.87-0.93)$ \\
\hline Model 1 & $0.95(0.92-0.98)$ & Model 1 & $0.90(0.87-0.93)$ \\
\hline Model 2 & $0.97(0.95-1.04)$ & Model 2 & $0.92(0.89-0.95)$ \\
\hline Model 3 & $0.98(0.94-1.00)$ & Model 3 & $0.92(0.89-0.95)$ \\
\hline Servings of whole grains & & Servings of coffee & \\
\hline Unadjusted & $0.95(0.94-0.97)$ & Unadjusted & $1.02(1.00-1.04)$ \\
\hline Model 1 & $0.95(0.94-0.97)$ & Model 1 & $1.02(1.00-1.03)$ \\
\hline Model 2 & $0.97(0.95-0.99)$ & Model 2 & $1.02(1.00-1.04)$ \\
\hline Model 3 & $0.97(0.96-0.99)$ & Model 3 & $1.02(1.00-1.04)$ \\
\hline Servings of refined grains & & Servings of decaffeinated coffee & \\
\hline Unadjusted & $1.09(1.07-1.11)$ & Unadjusted & $1.02(0.98-1.06)$ \\
\hline Model 1 & $1.08(1.06-1.10)$ & Model 1 & $1.03(0.99-1.08)$ \\
\hline Model 2 & $1.07(1.05-1.09)$ & Model 2 & $1.03(0.99-1.08)$ \\
\hline Model 3 & $1.06(1.05-1.07)$ & Model 3 & $1.04(1.00-1.09)$ \\
\hline Servings of milk products & & Servings of black tea & \\
\hline Unadjusted & $0.99(0.97-1.01)$ & Unadjusted & $1.00(0.98-1.02)$ \\
\hline Model 1 & $0.99(0.97-1.01)$ & Model 1 & $1.00(0.98-1.03)$ \\
\hline Model 2 & $0.99(0.97-1.01)$ & Model 2 & $1.00(0.98-1.02)$ \\
\hline Model 3 & $0.99(0.97-1.01)$ & Model 3 & $1.01(0.99-1.03)$ \\
\hline
\end{tabular}


Table 5. Cont.

\begin{tabular}{|c|c|c|c|}
\hline Variable & $\begin{array}{c}\text { Prevalence Ratio } \\
\text { (95\%CIs) }\end{array}$ & Variable & $\begin{array}{c}\text { Prevalence Ratio } \\
\text { (95\%CIs) }\end{array}$ \\
\hline Servings of eggs & & Servings of green tea & \\
\hline Unadjusted & $1.01(1.00-1.02)$ & Unadjusted & $0.97(0.94-1.01)$ \\
\hline Model 1 & $1.01(1.00-1.02)$ & Model 1 & $0.98(0.94-1.01)$ \\
\hline Model 2 & $1.01(1.00-1.02)$ & Model 2 & $0.99(0.96-1.03)$ \\
\hline Model 3 & $1.02(1.01-1.03)$ & Model 3 & $1.00(0.96-1.04)$ \\
\hline Servings of meat/poultry & & Servings of vegetables & \\
\hline Unadjusted & $1.06(1.05-1.07)$ & Unadjusted & $0.95(0.93-0.96)$ \\
\hline Model 1 & $1.06(1.05-1.07)$ & Model 1 & $0.95(0.93-0.96)$ \\
\hline Model 2 & $1.06(1.06-1.07)$ & Model 2 & $0.97(0.96-0.99)$ \\
\hline Model 3 & $1.05(1.04-1.06)$ & Model 3 & $0.97(0.96-0.99)$ \\
\hline Servings of fish & & Servings of fruit & \\
\hline Unadjusted & $1.03(0.99-1.06)$ & Unadjusted & $0.95(0.94-0.97)$ \\
\hline Model 1 & $1.03(1.00-1.07)$ & Model 1 & $0.95(0.94-0.97)$ \\
\hline Model 2 & $1.04(1.01-1.07)$ & Model 2 & $0.97(0.95-0.99)$ \\
\hline Model 3 & $1.04(1.01-1.07)$ & Model 3 & $0.97(0.95-0.99)$ \\
\hline Servings of tofu & & Servings of fruit juice & \\
\hline Unadjusted & $0.82(0.73-0.93)$ & Unadjusted & $0.99(0.97-1.01)$ \\
\hline Model 1 & $0.83(0.73-0.94)$ & Model 1 & $0.99(0.96-1.01)$ \\
\hline Model 2 & $0.84(0.74-0.96)$ & Model 2 & $1.00(0.97-1.02)$ \\
\hline Model 3 & $0.84(0.73-0.95)$ & Model 3 & $1.00(0.97-1.02)$ \\
\hline Servings of beans & & HEI & \\
\hline Unadjusted & $0.98(0.94-1.02)$ & Unadjusted & $0.99(0.99-0.99)$ \\
\hline Model 1 & $0.98(0.94-1.02)$ & Model 1 & $0.99(0.99-0.99)$ \\
\hline Model 2 & $1.00(0.96-1.05)$ & Model 2 & $1.00(0.99-1.00)$ \\
\hline Model 3 & $1.00(0.96-1.04)$ & Model 3 & $1.00(0.99-1.00)$ \\
\hline
\end{tabular}

Model 1, adjusted for sex and age; Model 2, adjusted for age, sex, education, smoking, alcohol use and physical activity; Model 3, adjusted for age, sex, education, smoking, alcohol use, physical activity and province. ${ }^{\dagger}$ Obesity is defined as a BMI $\geq 30.0 \mathrm{~kg} / \mathrm{m}^{2}$.

\section{Discussion}

Suboptimal diet quality has emerged as an important contributor to chronic disease [4-9]. In this study, we compared diet quality of adults in four Atlantic Canadian provinces and assessed the association between diet quality and obesity. Based on the regression models, participants were more likely to be obese if they consumed more meat/poultry, refined grains, soft drinks, snack foods, sweeteners, and visited fast food restaurants, and were less likely to be obese if they more frequently consumed garlic, hot spices and ginger, fruit and vegetables, whole grains, milk products, tofu, and nuts/seeds. Although these associations are not surprising, the differences in diet quality across the regions, as well as differences in measures of adiposity among provinces, highlights the need for distinct health policies and programs in each province to help combat the rising concern of obesity and related co-morbidities.

Regional studies have identified individual food trends and relationships between specific food items and disease. For example, there have been reported differences in sodium intake among people living in different areas of the United States [23] and, similar to the current study, a significant inverse relationship was observed between measures of adiposity (BMI and waist circumference) and whole grain consumption [24]. However, rather than focusing on individual food items, there has recently been a shift to exploring overall diet quality and the risk of disease or mortality [4-9].

Based on patterns of food intake, the American Heart Association has made recommendations for optimal cardiovascular health that focus on a combination of dietary components such as fruit and vegetables, fish, sodium, sugar-sweetened beverages, whole grains, nuts, seed and legumes, processed meat and saturated fat [25]. More specifically, excess sodium intake, processed meats, sugar sweetened beverages, and low intake of nuts/seeds, seafood omega-3 fats, vegetables, fruits and whole grains 
are associated with cardiometabolic deaths [4]. Importantly, the findings varied by sex, age, race, and education but, unfortunately, there were no region-specific data included.

Chen et al. identified four main dietary patterns in adults in Newfoundland and Labrador and showed that males were more likely to consume higher amounts of meat and fish whereas older participants were less likely to choose meat and more likely to choose fish [26]. Diet quality in the above study may be reflective of the history and culture of that specific coastal region which has traditionally been dependent on a fishing industry. Region-specific dietary data across a country are important for understanding factors that influence diet quality and developing policy, guidelines, and education programs to target those specific populations. When considering region-specific diet quality, it is also important to consider availability, particularly in rural/remote areas, and socio-demographic factors that influence diet quality. For example, household size and income influence food purchasing [27] and higher income and education levels are associated with higher fruit and vegetable consumption [28-30]. Furthermore, increased density of neighborhood fast-food restaurants was associated with unhealthy lifestyles such as low consumption of fruit and vegetables and not meeting physical activity guidelines [31]. Specifically, in Nova Scotia, the density of fast food restaurants has been associated with community-level material deprivation (social and/or material disadvantage) [32], an important factor that has been associated with larger waist circumference, waist-to-hip ratio, and BMI [33].

Few studies have investigated multiple measures of adiposity (BMI, waist circumference, waist-to-hip ratio, percent body fat) in relation to diet quality. Recently, the New Zealand Adult Nutrition Survey was used to examine the association between more than one adiposity measure (BMI and waist circumference) and diet quality [34]. In that study, an inverse association was reported between both BMI and waist circumference, and a "healthy" dietary pattern (characterized by high intake of breakfast cereal, low fat milk, soy and rice milk, soup and stock, yoghurt, fruit and tea, and low intakes of pies and pastries, potato chips, white bread, takeaway foods, soft drinks, beer and wine) compared to a "traditional" dietary pattern (characterized by high intake of beef, starchy vegetables, green vegetables, carrots, tomatoes, savory sauces, regular milk, cream, sugar, tea and coffee, and low intake of takeaway foods) [34]. A study conducted in Quebec, Canada demonstrated that participants within the highest Western-type diet tertile had the highest BMI, waist circumference, and fat mass; conversely, those in the highest Prudent-diet tertile had the lowest BMI, waist circumference, and fat mass [35]. In a national Canadian survey, higher diet quality was associated with a lower BMI and less obesity [36,37], however, it is known that access to different food sources varies [38] and consumption patterns are not equal across Canada [39]. The current study examined the distinct region of Atlantic Canada, which is known to have elevated levels of obesity and other chronic disease [2]. Findings demonstrate that those consuming low levels of fruits and vegetables, whole grains, nuts/seeds and higher levels of refined grains, meat/poultry, snack foods, sweeteners, soft drinks, and fast food were more likely to be obese.

The current study is unique in that it looks at diet quality in a specific region of the country and utilizes multiple measures of adiposity to explore relationships with dietary components. A major strength of the current study is that both measured and self-reported physical indices were used. Additionally, this study included participants from across the Atlantic region, including both rural and urban centers. However, the current study is not without limitations. For example, the dietary information was self-reported and hence subject to bias when participants are required to recall past dietary habits. Additionally, the current study utilizes an older method for calculating diet quality, however this method was chosen for comparison and consistency with earlier data analysis conducted on this cohort and the timeline of Atlantic PATH recruitment [22]. There are newer methods for calculating the HEI for the Canadian population [40], which should be considered for future studies. Finally, the study was cross-sectional in design, thus it can only explain associations, not causal effects. However, with the longitudinal design of the Atlantic PATH study, this type of analysis will be possible in the future with follow-up assessments in these participants. 
Future research should assess additional factors that may influence diet quality in a specific region, such as access and availability. Furthermore, due to geographic isolation and the importance of particular industries, it may be may be more relevant for certain areas to utilize specific dietary assessment tools [26]. Dietary questionnaires should also be expanded to include certain food items specific to the Canadian population which may positively or negatively influence obesity such as processed meats, cheese, gravies, sauces, and yogurt.

The study information will be useful for developing policy and strategies including food pricing, availability, and marketing strategies on food selection. It could also influence food guidelines and policies or programs to address needs of specific groups or populations. Public health policies in these regions need to limit the impact of detrimental food/nutrition environments and maximize access to healthy/positive food choices.

\section{Conclusions}

In conclusion, diet quality of participants living in the Atlantic region of Canada varied among provinces and were associated with multiple indices of obesity. These results should be used to help guide public health strategies aimed at altering dietary habits to improve health and reduce chronic disease.

Acknowledgments: The authors would like to acknowledge all the Atlantic PATH participants who donated their time, personal health history, and biological samples to this project as well as the Atlantic PATH team members for data collection, capture, and coding. This work was supported by the Canadian Partnership Against Cancer and Health Canada and they had no role in the design, analysis, or the writing of the article. The views expressed herein represent the views of the authors and do not necessarily represent the views of Health Canada.

Author Contributions: V.D. conceived the study, analyzed the data and wrote the manuscript. Y.C., T.D., C.F., S.G, M.K., L.P., E.S. and M.Y. substantially contributed to the interpretation of the data and critically revised for important intellectual content. All authors approved the final version of the manuscript.

Conflicts of Interest: The authors declare no conflict of interest.

\section{References}

1. Statistics Canada. Archived-Life Expectancy at Birth, by Sex, by Province. 2012. Available online: http: / / www.statcan.gc.ca/tables-tableaux/sum-som/101/cst01/hlth67-eng.htm (accessed on 24 July 2017).

2. Public Health Agency of Canada. How Healthy Are Canadians? A Trend Analysis of the Health of Canadians from a Healthy Living and Chronic Disease Perspective. 2016. Available online: https://www.canada.ca/content/dam/phac-aspc/documents/services/publications/healthy-living/ how-healthy-canadians/pub1-eng.pdf (accessed on 11 July 2017).

3. Statistics Canada. Health Indicator Profile, Annual Estimates, by Age Group and Sex, Canada, Provinces, Territories, Health Regions (2013 Boundaries) and Peer Groups, Occasional, CANSIM (Database). 2016. Available online: http:/ / www5.statcan.gc.ca/cansim/a26?lang=eng\&retrLang=eng\&id=1050501\& \&pattern=\&stByVal=1\&p1=1\&p2=37\&tabMode=dataTable\&csid=\#archived (accessed on 14 July 2017).

4. Micha, R.; Penalvo, J.L.; Cudhea, F.; Imamura, F.; Rehm, C.D.; Mozaffarian, D. Association between Dietary Factors and Mortality from Heart Disease, Stroke, and Type 2 Diabetes in the United States. JAMA 2017, 317, 912-924. [CrossRef] [PubMed]

5. Nanri, A.; Mizoue, T.; Shimazu, T.; Ishihara, J.; Takachi, R.; Noda, M.; Iso, H.; Sasazuki, S.; Sawada, N.; Tsugane, S.; et al. Dietary Patterns and all-Cause, Cancer, and Cardiovascular Disease Mortality in Japanese Men and Women: The Japan Public Health Center-Based Prospective Study. PLoS ONE 2017, 12, e0174848. [CrossRef] [PubMed]

6. Li, F.; Hou, L.N.; Chen, W.; Chen, P.L.; Lei, C.Y.; Wei, Q.; Tan, W.L.; Zheng, S.B. Associations of Dietary Patterns with the Risk of all-Cause, CVD and Stroke Mortality: A Meta-Analysis of Prospective Cohort Studies. Br. J. Nutr. 2015, 113, 16-24. [CrossRef] [PubMed]

7. Wirt, A.; Collins, C.E. Diet Quality-What is it and does it Matter? Public Health Nutr. 2009, 12, $2473-2492$. [CrossRef] [PubMed] 
8. Kant, A.K. Dietary Patterns: Biomarkers and Chronic Disease Risk. Appl. Physiol. Nutr. Metab. 2010, 35, 199-206. [CrossRef] [PubMed]

9. Schwingshackl, L.; Hoffmann, G. Diet Quality as Assessed by the Healthy Eating Index, the Alternate Healthy Eating Index, the Dietary Approaches to Stop Hypertension Score, and Health Outcomes: A Systematic Review and Meta-Analysis of Cohort Studies. J. Acad. Nutr. Diet. 2015, 115, 780-800. [CrossRef] [PubMed]

10. GBD 2015 Obesity Collaborators; Afshin, A.; Forouzanfar, M.H.; Reitsma, M.B.; Sur, P.; Estep, K.; Lee, A.; Marczak, L.; Mokdad, A.H.; Moradi-Lakeh, M.; et al. Health Effects of Overweight and Obesity in 195 Countries Over 25 Years. New Engl. J. Med. 2017, 377, 13-27. [CrossRef] [PubMed]

11. Heymsfield, S.B.; Wadden, T.A. Mechanisms, Pathophysiology, and Management of Obesity. New Engl. J. Med. 2017, 376, 254-266. [CrossRef] [PubMed]

12. Hruby, A.; Manson, J.E.; Qi, L.; Malik, V.S.; Rimm, E.B.; Sun, Q.; Willett, W.C.; Hu, F.B. Determinants and Consequences of Obesity. Am. J. Public Health 2016, 106, 1656-1662. [CrossRef] [PubMed]

13. Asghari, G.; Mirmiran, P.; Yuzbashian, E.; Azizi, F. A Systematic Review of Diet Quality Indices in Relation to Obesity. Br. J. Nutr. 2017, 117, 1055-1065. [CrossRef] [PubMed]

14. Guenther, P.M.; Casavale, K.O.; Reedy, J.; Kirkpatrick, S.I.; Hiza, H.A.; Kuczynski, K.J.; Kahle, L.L.; Krebs-Smith, S.M. Update of the Healthy Eating Index: HEI-2010. J. Acad. Nutr. Diet. 2013, 113, 569-580. [CrossRef] [PubMed]

15. Garriguet, D. Diet Quality in Canada. Health Rep. 2009, 20, 41-52. [PubMed]

16. Health Canada. Eating Well with Canada's Food Guide. 2011. Available online: https: / / www.canada.ca/content/dam/hc-sc/migration/hc-sc/fn-an/alt_formats/hpfb-dgpsa/pdf/foodguide-aliment/print_eatwell_bienmang-eng.pdf (accessed on 11 July 2017).

17. Statistics Canada. Overweight and Obese Adults (Self-Reported). 2014. Available online: http://www. statcan.gc.ca/pub/82-625-x/2015001/article/14185-eng.htm (accessed on 14 July 2017).

18. International Physical Activity Questionnaire Group. International Physical Activity Questionnaire. 2002. Available online: http://www.sdp.univ.fvg.it/sites/default/files/IPAQ_English_self-admin_long.pdf (accessed on 8 August 2017).

19. International Physical Activity Group. Guidelines for Data Processing and Analysis of the International Physical Activity Questionnaire (IPAQ). 2005. Available online: https://sites.google.com/site/theipaq/ scoring-protocol (accessed on 8 August 2017).

20. Schutz, Y.; Kyle, U.U.; Pichard, C. Fat-Free Mass Index and Fat Mass Index Percentiles in Caucasians Aged 18-98 Y. Int. J. Obes. Relat. Metab. Disord. 2002, 26, 953-960. [CrossRef] [PubMed]

21. Grundy, S.M.; Cleeman, J.I.; Daniels, S.R.; Donato, K.A.; Eckel, R.H.; Franklin, B.A.; Gordon, D.J.; Krauss, R.M.; Savage, P.J.; Smith, S.C., Jr.; et al. Diagnosis and Management of the Metabolic Syndrome: An American Heart Association/National Heart, Lung, and Blood Institute Scientific Statement. Circulation 2005, 112, 2735-2752. [CrossRef] [PubMed]

22. Yu, Z.M.; Parker, L.; Dummer, T.J. Depressive Symptoms, Diet Quality, Physical Activity, and Body Composition among Populations in Nova Scotia, Canada: Report from the Atlantic Partnership for Tomorrow's Health. Prev. Med. 2014, 61, 106-113. [CrossRef] [PubMed]

23. Harnack, L.J.; Cogswell, M.E.; Shikany, J.M.; Gardner, C.D.; Gillespie, C.; Loria, C.M.; Zhou, X.; Yuan, K.; Steffen, L.M. Sources of Sodium in US Adults from 3 Geographic Regions. Circulation 2017, 135, 1775-1783. [CrossRef] [PubMed]

24. Albertson, A.M.; Reicks, M.; Joshi, N.; Gugger, C.K. Whole Grain Consumption Trends and Associations with Body Weight Measures in the United States: Results from the Cross Sectional National Health and Nutrition Examination Survey 2001-2012. Nutr. J. 2016, 15. [CrossRef] [PubMed]

25. Mozaffarian, D.; Benjamin, E.J.; Go, A.S.; Arnett, D.K.; Blaha, M.J.; Cushman, M.; de Ferranti, S.; Despres, J.P.; Fullerton, H.J.; Howard, V.J.; et al. Heart Disease and Stroke Statistics-2015 Update: A Report from the American Heart Association. Circulation 2015, 131, e29-e322. [CrossRef] [PubMed]

26. Chen, Z.; Liu, L.; Roebothan, B.; Ryan, A.; Colbourne, J.; Baker, N.; Yan, J.; Wang, P.P. Four Major Dietary Patterns Identified for a Target-Population of Adults Residing in Newfoundland and Labrador, Canada. BMC Public Health 2015, 15. [CrossRef] [PubMed]

27. Ricciuto, L.; Tarasuk, V.; Yatchew, A. Socio-Demographic Influences on Food Purchasing among Canadian Households. Eur. J. Clin. Nutr. 2006, 60, 778-790. [CrossRef] [PubMed] 
28. Tarasuk, V.; Fitzpatrick, S.; Ward, H. Nutrition Inequities in Canada. Appl. Physiol. Nutr. Metab. 2010, 35, 172-179. [CrossRef] [PubMed]

29. Rehm, C.D.; Penalvo, J.L.; Afshin, A.; Mozaffarian, D. Dietary Intake among US Adults, 1999-2012. JAMA 2016, 315, 2542-2553. [CrossRef] [PubMed]

30. Dehghan, M.; Akhtar-Danesh, N.; Merchant, A.T. Factors Associated with Fruit and Vegetable Consumption among Adults. J. Hum. Nutr. Diet. 2011, 24, 128-134. [CrossRef] [PubMed]

31. Li, F.; Harmer, P.; Cardinal, B.J.; Bosworth, M.; Johnson-Shelton, D. Obesity and the Built Environment: Does the Density of Neighborhood Fast-Food Outlets Matter? Am. J. Health Promot. 2009, 23, 203-209. [CrossRef] [PubMed]

32. Jones, J.; Terashima, M.; Rainham, D. Fast Food and Deprivation in Nova Scotia. Can. J. Public Health 2009, 100, 32-35. [PubMed]

33. Ellaway, A.; Anderson, A.; Macintyre, S. Does Area of Residence Affect Body Size and Shape? Int. J. Obes. Relat. Metab. Disord. 1997, 21, 304-308. [CrossRef] [PubMed]

34. Beck, K.L.; Jones, B.; Ullah, I.; McNaughton, S.A.; Haslett, S.J.; Stonehouse, W. Associations between Dietary Patterns, Socio-Demographic Factors and Anthropometric Measurements in Adult New Zealanders: An Analysis of Data from the 2008/09 New Zealand Adult Nutrition Survey. Eur. J. Nutr. 2017. [CrossRef] [PubMed]

35. Paradis, A.M.; Godin, G.; Perusse, L.; Vohl, M.C. Associations between Dietary Patterns and Obesity Phenotypes. Int. J. Obes. 2009, 33, 1419-1426. [CrossRef] [PubMed]

36. Jessri, M.; Wolfinger, R.D.; Lou, W.Y.; L'Abbe, M.R. Identification of Dietary Patterns Associated with Obesity in a Nationally Representative Survey of Canadian Adults: Application of a Priori, Hybrid, and Simplified Dietary Pattern Techniques. Am. J. Clin. Nutr. 2017, 105, 669-684. [CrossRef] [PubMed]

37. Sundararajan, K.; Campbell, M.K.; Choi, Y.H.; Sarma, S. The Relationship between Diet Quality and Adult Obesity: Evidence from Canada. J. Am. Coll. Nutr. 2014, 33, 1-17. [CrossRef] [PubMed]

38. Smoyer-Tomic, K.E.; Spence, J.C.; Raine, K.D.; Amrhein, C.; Cameron, N.; Yasenovskiy, V.; Cutumisu, N.; Hemphill, E.; Healy, J. The Association between Neighborhood Socioeconomic Status and Exposure to Supermarkets and Fast Food Outlets. Health Place 2008, 14, 740-754. [CrossRef] [PubMed]

39. Statistics Canada, G.D. Overview of Canadians Eating Habits. 2004. Available online: http:/ / www.statcan. gc.ca/pub/82-620-m/82-620-m2006002-eng.pdf (accessed on 24 July 2017).

40. Jessri, M.; Ng, A.P.; L'Abbé, M.R. Adapting the Healthy Eating Index 2010 for the Canadian Population: Evidence from the Canadian National Nutrition Survey. Nutrients 2017, 910. [CrossRef] 